\title{
The Influence Factors of Energy Efficiency in China Based on Meta- Regression
}

\author{
Yang Yue, Pan Xiongfeng ${ }^{*}$ and Liu Qing \\ Dalian University of Technology, Faculty of Management and Economics, 116023 Dalian, P.R. China
}

\begin{abstract}
This paper is based on the controversies which the existing empirical researches on the impact of the basic factors affecting energy efficiency, have in index selection and direction, using the method of meta-regression to analyze the related domestic empirical studies comprehensively. We conducted a study on the differences of research design, data characteristics, time span and the index selection, to check whether these differences cause the controversies in index selection and direction. The result shows that the research design, empirical methods, and the characteristics of the selection of indicators have significant impact on the result of empirical researches.
\end{abstract}

Keywords: Energy efficiency, Influence factors, Meta-regression, Empirical researches, Energy revolution, T-statistic values, Domestic empirical studies.

\section{INTRODUCTION}

The contradiction between higher energy consumption and lower energy efficiency makes energy shortage more and more serious. In 2010, the World Bank in its "Report of the development level of human society" considered the energy efficiency as one of the key indicators to measure the progress of human society and economic development. As the developing countries face the challenge of economic growth resulted by energy shortages and energy crises, the 18th of the National People's Congress pointed out to control energy consumption, and used "Energy Revolution" to reveal the importance and seriousness of the energy efficiency. Thus, the efficient use of energy has become a hot topic for scholars who are competing for studying, then confirming the influence factor on energy efficiency and finding out the interaction mechanism of these factors which will become the starting point to solve the problem of lower energy efficiency. The researches in this field are rather rich.

Shi Dan (2002) pointed out that opening up, industrial structure and economic system are the important factors affecting energy efficiency [1]. Paul Crompton (2005) and Fisher-Vanden (2004) analyzed the influencing factors of energy efficiency of China, and they regarded that the technological progress, industrial restructuring and energy prices are the driving forces to improve energy efficiency [2]. In addition, Fisher considered energy consumption structure as an important factor for energy efficiency, and an important factor leading to China's energy efficiency and energy intensity reduction is the continuous reduction of coal consumption proportion in the industrial sector [3]. Pan Guogang and Jia Jiangtao (2012) sorted out that structure, technology, marketing and human are the four factors affecting energy efficiency [4]. Yu Jianxun (2011) used

*Address correspondence to this author at the Dalian University of Technology, Faculty of management and Economics, 116023 Dalian, P.R. China; E-mail: xiongfengpan@dlut.edu.cn stepwise regression method to test the influences on energy efficiency of these three factors: economic growth, science and technology investment, and the degree of economic openness [5]. Tan Zhongfu and Zhang Jinliang (2010) selected the economic structure, technological progress, energy consumption structure and energy prices to establish state-space model of energy efficiency [6]. Xia Ling (2012) and Han Lu (2011) both chose Tobit model to test the influence factors of energy efficiency, but the indicators they selected were different [7, 8]. Yang Mian and Yang Fuxia used generalized impulse response function to conduct the empirical study [9]. However, most empirical researches are flawed, due to the emphasis on a different angle, or simply because the conclusions are not significant enough to eliminate a few factors, which lead to the lack of a comprehensive, standardized and systematic measurement system.

Qu Xiaoe (2009) made the conclusion that technical progress has a positive impact on improving energy efficiency [10]. By contrast, Li Jiyang (2011) thought the effect of technical progress is not significant [11]. Chen Xiaoyi (2012) proved that when the value of energy efficiency was low, technological progress would promote energy efficiency, but when energy efficiency was high, technological progress exerted a "Rebound Effect" [12]. While Li Guozhang (2009) and Li Jiyang (2011) believed that the openness had a significant positive effect [13]. On the contrary, Sun Jingshui (2011) and Hu Zongyi (2011) concluded that the openness had a negative effect $[14,15]$. Based on these conclusions, Wei Chu and Shen Manhong insisted that the openness had a dual effect on energy efficiency; on one hand, it improved the energy efficiency of the region by the introduction of advanced technology, equipment and management experience; on the other hand, foreign direct investment and multinational technical levels exacerbated the differences between the various regional companies, thereby reducing the entire region's relative average frontier [16]. In fact, the disagreement and confusion is related to the selection of influence factors, different 
empirical studies had differences on the sample data, modeling design and estimation methods, which would lead to different empirical results.

In view of the shortcomings and defects on previous analyses of energy efficiency factors, this paper attempts to apply the method of meta-regression to make reanalysis of factors affecting energy efficiency. This empirical study finds out the energy efficiency aspects to a significant degree or even a sign above the ambiguity, through simple qualitative description by collecting previous literature regarding certain rules on energy efficiency factors, and explains these influencing factors comprehensively and systematically.

\section{MATERIALS AND METHODOLOGY}

\subsection{Materials}

Compared with the traditional methods, the metaregression analysis (MRA) is a quantitative and systematic research method, which can integrate a number of empirical research findings with common purposes, to achieve a twostage model summarizing certain research areas. It is not limited to traditional qualitative description and summary of literature review, preventing the subjective preferences of the authors to a large extent. More importantly, it is able to explore the use of information contained in previous literature and verify the deep-seated reasons of differences in the conclusions of previous studies, namely to find the effect of adjusting the variables and testing of the manipulated variables. (Stanley, 2001; Higgins and Thompson, 2002).

In the model of meta-regression, the explained variable is the estimated value of a variable's $(X)$ influence coefficient $(\beta)$ to another variable $(\mathrm{Y})$ or t-statistic; the explanatory variables are extracted from previous studies representing the characteristics of a range of factors, such as sample composition, model design, estimation methods, and the properties of authors and their institutions (Stanley, 2001; Stanley and Doucouliagos, 2009) $[17,18]$. If the result of an explanatory variable in meta-regression is significant, it means that the characteristics represented by this variable affect the previous literature results significantly on the conclusion of "X for Y influence". Meta-regression can be described as:

$$
Y_{\mathrm{j}}=\beta_{0}+\sum_{k=1}^{K} \beta_{k} Z_{\mathrm{jk}}+e_{j}, j=1,2 \ldots \ldots . N
$$

In Equation (1), the explained variable $\mathrm{Yj}$ is the t-statistic of parameter estimates in general, the value of t-statistic is derived from an empirical study with $\mathrm{N}$ conclusions, and $\mathrm{N}$ is the sample size of meta-regression. $Z j k(k=1,2 \ldots \ldots . \mathrm{K})$ in the meta-regression is the proxy variable with the number of $\mathrm{K}$, reflecting all of the differences in the selection of sample data, model design, and estimation methods.

\subsection{Methodology}

\subsubsection{The Selection of Sample}

This paper using Chinese Journal Full-text database, searching "topic", "abstract", "keyword" with the key words "energy efficiency" and "impact factor", collected all the literature on factors affecting energy efficiency in China. We got 36 papers which meet the requirements, after a preliminary filtering and organization. From these 36 empirical papers, we extracted the information including: author, published publications, publishing time, data relating to the life of the sample used, the type of sample data (crosssectional data, time series data or panel data), and the level of the sample data (the national level data, inter-provincial data, industry data or micro-data on enterprise level). Because all of the papers are published to the public, and working papers are not included in the sample, this paper will not report the publication bias. This discrimination usually refers to some specific type or papers with specific conclusion that are often easier to get published, or a higherlevel magazine that tends to get some papers published with specific conclusions.

This paper finally selected 79 copies of conclusions from the 36 empirical papers, and the way of selection we chose was learned from Wang Wanjun [19]. If a paper appears with multiple models and empirical descriptions, this paper will select the best estimate of the results as the authors believed. If the authors do not point out the most preferred empirical conclusions, yet the results of the values and symbols between different method estimates are not significantly difference, this paper will select the one with the highest goodness of fit. When different explained variables, important explanatory variables and estimation methods are replaced in the paper, or using a different sub-sample data, or contain a different measure for a variable, while not taking a certain test method to point out the best estimation results, all of the same paper's conclusions will be selected at the same time.

In the 79 results based on experience, the distribution and status of t-statistics values of the number of the main factors studied in this paper, are shown in Table 1. We made the following conclusion: in addition to the structure of foreign trade, the empirical results of other factors have considerable differences. Worth noting is that we found that the problem of symbol is due to some scholars using the proportion of secondary industry as a measure of industrial structure, as described before, so their impact on energy efficiency will be negative. However, some scholars have taken the proportion of tertiary industry as the measure, thus the conclusions will be exactly the opposite, while their impact on energy efficiency is positive. Therefore, since the differences based on symbols do not exist essentially, so we analyzed why the results are not significant instead of the difference of symbols.

\subsubsection{Variables}

The description of explanatory variables included in metaregression is given in Table 2 . The explained variable is tstatistic values of i-th parameter estimators for energy efficiency. When estimating the parameters of energy efficiency in empirical research, the corresponding parameter estimates standard deviation or t-statistic values will be given. Thus, if the results of empirical studies do not report the value of $\mathrm{t}$ statistic, we can calculate $t$-statistic values based on parameter estimates and the corresponding standard deviation. This means 
Table 1. The significant distribution of main influence factors.

\begin{tabular}{|c|c|c|c|c|c|c|c|c|}
\hline \multirow{2}{*}{ Variables } & \multirow{2}{*}{ Total } & \multicolumn{3}{|c|}{ The Number of Occurrences } & \multirow{2}{*}{ Mean } & \multirow{2}{*}{ Sd } & \multicolumn{2}{|c|}{$\mathbf{t}$} \\
\hline & & Positive & Not Significant & Negative & & & Min & Max \\
\hline Economic level & 25 & 8 & 9 & 8 & -2.453 & 0.8911 & -159 & 3.182 \\
\hline Industrial Structure & 79 & 36 & 28 & 15 & 0.502 & 0.7404 & -38 & 12.097 \\
\hline Opening & 31 & 22 & 8 & 1 & 0.018 & 0.025 & -4.058 & 10.009 \\
\hline Energy consumption & 59 & 17 & 14 & 28 & -1.443 & 0.586 & -10.77 & 17 \\
\hline Technical progress & 69 & 48 & 11 & 10 & 0.143 & -0.072 & -67 & 21.01 \\
\hline Energy prices & 57 & 40 & 11 & 6 & 0.204 & 0.071 & -34.31 & 18.851 \\
\hline Institutional factors & 30 & 22 & 1 & 7 & 1.922 & 0.587 & -11.04 & 18.573 \\
\hline
\end{tabular}

Table 2. The variables included in meta-regression.

\begin{tabular}{|c|l|}
\hline Variables & \\
\hline \hline Yi & t-statistic values of influence variables for energy efficiency \\
\hline varnum & the number of variables selected in the research \\
\hline year & the indicator of time span selected in the research \\
\hline $1 /$ se & the reciprocal of standard error of each variable \\
\hline Ystyle & If we choose the TFP method, the value is $1 ;$ if the method is single factor, the value is 0. \\
\hline Panel & If it is a panel data, the value is $1 ;$ if it is time data or sectional data, the value is 0. \\
\hline Tobit & If the method of regression is Tobit, the value is $1 ;$ otherwise, it is 0. \\
\hline stepwise & If chosen stepwise regression to select variables before setting up the model, the value is $1 ;$ otherwise, it is 0. \\
\hline dynamic & If using dynamic model, that is to say the independent variables contains lagged terms, the value is $1 ;$ otherwise, it is 0. \\
\hline fe & If fixed effects model is used, the value is $1 ;$ otherwise, it is 0. \\
\hline insform & If it is a measure of growth rate of the industrial structure, the value is $1 ;$ if it is only the proportion, the value is 0. \\
\hline tecform & If it is a relative amount of technical progress, the value is $1 ;$ if it is an absolute amount, the value is 0. \\
\hline epform & If it is a comprehensive measure of the price index of Energy prices, the value is $1 ;$ otherwise, it is 0. \\
\hline
\end{tabular}

that if we cannot get the value of t-statistic, the corresponding papers will not select meta-regression.

Explanatory variables describe the characteristics of sample data, model design, and estimation methods in the empirical research. "Ystyle" describes the explained variables as how to define a dummy variable. When energy efficiency is calculated by TFP with DEA method, "Ystyle" equals to 1 , and when energy efficiency is chosen to represent the energy consumption per unit of GDP, Y equals to 0 . Then, "Tobit", "stepwise", "dynamic" and "fe" are the dummy variables which explain the regression in this empirical study. Since the efficiency values obtained from DEA method for each region, are truncated data, and using OLS to estimate the parameters may be biased, some scholars choose Tobit model to solve this problem. At the same time, "stepwise" describes the process of variables selection, i.e. if the estimation before setting up the formal modeling uses stepwise regression to select variables, the value is 1 , otherwise it is 0 . Due to the impact of various factors on energy efficiency, lag effect may exist, so "dynamic" is included in the study to distinguish whether it is in consideration. "fe" is used to distinguish whether
Hausman test is done when setting the model, in order to determine which is more suitable between the Fixed Effect and Random Effect.

As explanatory variables in the meta-regression, the dummy variables "insform" and "tecform" are used to distinguish the selection of influence factors in different literatures. When measure industrial structure optimization, if only consider the secondary and tertiary industry accountted for the proportion of GDP, then it only describes the expression of the industrial structure; and if using the proportion of secondary and tertiary industries in the incremental of total output, the effect of industrial structure adjustment will be considered as a whole. "tecform" means that if we adopt the relative variables, such as labor productivity, the proportion of foreign investment accountting for total investment, total factor productivity and the proportion of number of R\&D investment accounted for total sales when selecting the indicators of technological progress, then "tecform" equals to 1 ; if we select the absolute variables such as the number of granted patents, R\&D investment, etc., then "tecform" equals to 0 . 
As explanatory variables, "Year", "Panel" and "Varnum" in meta-regression analysis are used to describe the characteristics of the sample data. "year" is the indicator of time span selected in the sample data. "Panel" is a dummy variable, and if it is a panel data, the value is 1 ; if it is time data or sectional data, the value is 0 . "Varnum" is the number of variables selected in this research.

\section{RESULTS}

In order to make the meta-regression analysis more comprehensive, we used "metareg" analysis proposed by the Higgins and Thompson (2008) [20], conducting metaregression for each of the six factors. The advantage of this method is that it can be used in small number of experiments, and can adjust multiple tests when there are multiple covariates with permute option. Using Stata12 to finish the process, the results are shown in Table 3.

From the results of meta-regression, we can draw conclusion that the characteristics of research design and the selection of empirical method do have significant impact on the empirical results.

Firstly, the corresponding estimation results of "ystyle" are positive numbers in addition to the economic level and energy consumption structure, and all of them have passed the significance testing. This means that when analyzing the influence factors of energy efficiency, using the TPF method to calculate the energy efficiency, compared with the single element, has a more significant positive impact. When the coefficient is negative, it indicates that choosing TFP method for measuring energy consumption structure will get lower effect value, because the influence of the energy consumption structure on energy efficiency itself is negative. This has been analyzed in the theoretical part, so that we can get the following conclusion: the method of TFP is easier to get significant results from variables.

Secondly, the corresponding estimation results of "panel" and "dataform" are negative numbers in addition to some individual factors, and most of them have passed the significance testing, which means that using the panel data has a more significant result compared with time data and cross-sectional data; using provincial level data is often estimated to exert a higher positive effect than industry level and firm-level data, Often, the results of the coefficient to energy prices is negative, which passed the significant test. This indicates that using provincial-level data of energy price has a weaker impact on energy efficiency than using the industry level and firm-level data.

Thirdly, corresponding coefficients of "dynamic" are positive, indicating that if we consider the lag factor, compared with the non-dynamic model, the effect of factors tends to be higher. Fourthly, coefficients of "stepwise" and "fe" are negative, and half have passed the significance test, which shows that when using a stepwise regression model based on variable selection or fixed effects selection, compared with the literature that did not use this method, influencing factors effect is lower. Fifth, corresponding coefficient symbols of "year" are not regular, and in addition to the positive impact of technological progress, are significant. The other factors also passed significance testing, which means if the results are remarkable, they have no obvious relationship with time span of the samples; technological progress is likely to need a period of time to put an effect on energy efficiency, so a larger time span of sample would have a more significant result to some extent. Sixth, Tobit factor analysis shows that the economic level and opening using Tobit model will bring a higher level of impact, and the industrial structure, the structure of energy consumption and the impact of energy prices will lead to lower impact. Seventh, all the "varnum" don't pass the significance test, indicating the number of factors have no impact on the results obviously.

In addition, the different form of variables will also exert significant effect on the results. The coefficients of "Insform" are positive and have passed significance test, which means using the proportion of the second and tertiary industrial added value accounted for total output increment value; that is to say, the use of growth rate will give more significant results compared with using absolute variables.

Table 3. The results of meta--regression.

\begin{tabular}{|c|c|c|c|c|c|c|}
\hline Ystyle & $-15.128(-1.55)$ & $5.217 * *(2.00)$ & $1.059(0.64)$ & $-3.115(-1.65)$ & $20.395 * * *(4.11)$ & $16.095 * *(2.04)$ \\
\hline Panel & $388.067 *(1.96)$ & $5.833 * * *(2.39)$ & $3.794(0.67)$ & $4.441 *(1.79)$ & $24.285^{* * *}(5.59)$ & $-7.218(-1.14)$ \\
\hline dataform & $368.391 *(2.08)$ & $4.066(1.17)$ & - & - & $12.677 * *(2.57)$ & $-27.657 * * *(-2.95)$ \\
\hline varnum & $-0.695(-1.14)$ & $1.547(1.26)$ & $-0.012(-0.01)$ & $-1.306(-0.89)$ & $-2.821(-1.42)$ & $1.279(0.43)$ \\
\hline year & $3.909(1.22)$ & $-0.5707(-0.68)$ & $-0.074(-0.1)$ & $-1.04(-1.28)$ & $5.663 * * *(4.26)$ & $-3.584 *(-1.88)$ \\
\hline stepwise & $-6.553(-0.76)$ & $-12.663 * * *(-4.23)$ & $-0.339(-0.06)$ & $18.625 * * *(4012)$ & $-39.068 * * *(-9.6)$ & $14.787(1.38)$ \\
\hline dynamic & $362.399 *(1.95)$ & $1.619(0.44)$ & $13.726^{* *}(2.1)$ & - & - & - \\
\hline fe & $-0.972(-0.35)$ & $-11.697 * * *(-3.96)$ & $3.477(0.9)$ & $5.198 *(1.76)$ & $-38.919 * *(-8.75)$ & $-3.220(-0.5)$ \\
\hline $1 / \mathrm{se}$ & $0.229(1.15)$ & $-0.016 * * *(-4.55)$ & $-0.00008(-0.26)$ & $-0.0001(-0.24)$ & $-0.0001 * * *(-5.72)$ & $0.001(1.1)$ \\
\hline insform & - & $4.464 *(1.9)$ & - & - & - & - \\
\hline obs & 78 & 78 & 31 & 59 & 60 & 57 \\
\hline
\end{tabular}




\section{CONCLUSION}

A number of scholars have undertaken a lot of research on the impact of energy efficiency factors, but there is still disagreement on the selection of the influence factors and impact direction of these affecting factors on energy efficiency. It also failed to form a comprehensive and systematic index system. In fact, the differences in empirical research can be attributed to the differences in sample selection, model design and estimation methods of the study to a certain extent. Meta-regression analysis is a quantitative method of literature study, which can be used to analyze the correlation between the empirical study of an important parameter and design features of the study itself.

This paper breaks through the traditional method of literature review, using meta-regression analysis, keeping the empirical results of 79 of the 33 papers filtered for quantitative, systematic and accurate grooming, and analyses specific factors one by one. Explained variable of metaregression is the value of t-statistic of energy efficiency effect, thus the symbol of t-statistic values passed significance testing to a certain confidence level directly responding to the impact on the conclusions of empirical research. Explanatory variables of meta-regression describe the feature of sample data selection, model design, estimation methods and other aspects of the empirical research, so that eventually we obtained the following conclusions:

First, selecting the sample data will affect the empirical research conclusions on energy efficiency factors. Compared to the cross-sectional data or time-series data, selecting the panel data for empirical studies tends to obtain a higher effect; spillovers effect is stronger when selecting the provincial level than the industry derived data. Secondly, different estimation methods also affect the conclusions of empirical research; the impact effect of Tobit is more significant than other estimation methods (such as OLS and GLS). If conducting a selection of variables by stepwise regression method in empirical research, it tends to obtain a lower impact than those without filtering; Whether the model considers the lag factor will also have significant impact on results. Finally, explained and explanatory variables defined in the empirical study will also have some impact on the estimation results. For example, the explained variable of estimated model using Total Factor variable selection method for calculating energy efficiency will have a more significant impact on the results compared with the single factor method. Using the proportion of the second and tertiary industrial added value accounted for total output increment value, that is to say the use of growth rate will give more significant results compared with using absolute variables.

\section{CONFLICT OF INTEREST}

The authors confirm that this article content has no conflict of interest.

\section{ACKNOWLEDGEMENTS}

This paper is the stage achievement of the Liaoning Social Science Fund Project (L13DGL033) and National Nature Science Foundation Project (71303029). The author thanks for the support of The Liaoning Social Science Foundation and National Nature Science Foundation.

\section{REFERENCES}

[1] Dan, S. The improvement of energy consumption efficiency in China's economic growth. Econ. Res. J., 2002, 9, 49-94.

[2] Poul, C.; Wu, Y. Energy consumption in China past trends and future directions. Energy Econ., 2005, 27(1), 195-208.

[3] Fisher-Vanden, K. What is driving China's decline in energy intensity? Resour. Energy Econ., 2004, 26(1), 77-97.

[4] Pan, G.; Tao, J.J. Analysis of energy efficiency's influential factors. Energy Conserv. Technol., 2012, 1. 76-87

[5] Yu, J. Empirical analysis of factors influencing energy efficiency. Guide Bus., 2011, 2, 1-3

[6] Tan, Z.; Zhang, J. Demonstrational analysis of the relationship between energy efficiency and its influential factor in China. China Popul. Resour. Environ., 2010, 4, 43-49.

[7] Xia, L.; Fan, H.; Wu, W.; Zhu, B. The influence factors analysis of Chinese provincial energy efficiency based on DEA-Tobit model. J. Wuyi Univ., 2012, 4, 47-71.

[8] Han, L.; Yu, Y.; Song, M. Regional energy efficiency evaluation and its influencing factors. China Nat. Cond. Strength, 2011, 8, 1318

[9] Yang, M.; Yang, F.; Chen, X. On influencing factors affecting China's energy efficiency: an empirical test based on the VEC model. Res. Sci., 2011, 1, 163-168

[10] Qu, X. An analysis on Chinese inter-provincial differences in energy efficiency and their determinants. Econ. Theory Business Manage., 2009, 2, 46-52.

[11] Li, J. Empirical analysis on influencing factor of Chinese provincial energy efficiency. J. Xiangtan Univ., 2011, 4, 53-57.

[12] Chen X. A demonstrational analysis of influence factor on China's energy efficiency based on the quantile regression model. Econlog. Econ., 2012, 8, 63-66.

[13] Li, G.; Huo, Z. Chinese total factor energy efficiency, convergence and influencing factors-based on 1995-2006 An empirical analysis of provincial panel data. Econ. Rev., 2009, 6, 101-109

[14] Sun, J.; Wang, D. An analysis on Chinese district differences in energy efficiency and their determinants. Technol. Econ. Manag. Res., 2011, 12, 77-81.

[15] Hu, Z.; Liu, J.; Liu, Y. Research on inter-provincial differences in energy efficiency of China and its influencing factors. China Popul. Res. Environ., 2011, 7, 33-39.

[16] Wei, C.; Shen, M. Energy efficiency and its impact factors: an empirical analysis based on DEA. Manag. World, 2007, 8, 66-76.

[17] Higgins, J.; Thompson, S.G. Quantifying heterogeneity in a metaanalysis. Stat. Med., 2002, 21(11), 1539-1558.

[18] Doucouliagos, H.; Stanley, T. Publication selection bias in minimum wage research? A meta regression analysis. Br. J. Indust. Relat., 2009, 47(2), 406-428.

[19] Wang, W. The spillover effect of FDI in China: A re-analysis based on meta regression analysis. Econ. Rev., 2010, 1, 18.

[20] Harbord, R.M.; Higgins, J.P.T. Meta-regression in stata. Stat J., 2008, 8(4), 493-519. 